\title{
Why Does No One Want to Perform Lymph Node Dissection Anymore?
}

\author{
Kelly M. McMasters, MD, PhD \\ Department of Surgery, University of Louisville School of Medicine, James Graham Brown Cancer Center, Louisville, KY
}

The truth is rarely pure and never simple.

Oscar Wilde

It is error only, and not truth, that shrinks from inquiry.

Thomas Paine

In seeking absolute truth we aim at the unattainable and must be content with broken portions.

William Osler

In this issue of Annals of Surgical Oncology, Kingham et al. from Memorial Sloan-Kettering Cancer Center present their single-institution retrospective experience of melanoma patients who did not undergo completion lymph node dissection (CLND) for a tumor-positive sentinel lymph node (SLN). ${ }^{1}$ Of 313 patients who had a tumorpositive SLN, 271 underwent CLND and 42 did not. Of the 42 patients who did not undergo CLND, 5 did not do so because they were found to have stage IV disease on further workup, leaving 37 patients who should reasonably have considered CLND. The nodal recurrence rates were no different in the no-CLND group (7\%) versus the CLND group $(6 \%)$. Because the disease-free and disease-specific survival rates were similar between the groups, the authors question the value of CLND.

We must recognize the limitations of this study. It is a small, retrospective review from a single institution. The groups are not well balanced in terms of clinicopathologic factors. Patients in the no-CLND group versus the CLND group were older (median age, 70 vs. 56 years, $P<.01$ ), more often had lower-extremity melanomas ( $40 \%$ vs. $13 \%$,

(C) Society of Surgical Oncology 2009

Published Online: 26 November 2009

K. M. McMasters, MD, PhD

e-mail: kelly.mcmasters@ nortonhealthcare.org
$P<.01)$, and had a trend toward thicker (3.5 vs. $2.8 \mathrm{~mm}$, $P<.06$ ), more often ulcerated (62 vs. $44 \%, P=.09$ ) melanomas. ${ }^{1}$ Why did the patients not undergo CLND in this study? In $33 \%$ of cases, it seems that the surgeon essentially talked the patient out of having a CLND. Although patient refusal was cited as the most common reason for failure to perform CLND (45\%), it is likely that the surgeon's opinion strongly influenced the patients' decisions in these cases as well. The patients who chose to forego CLND did not do so for random reasons; selection bias is a major drawback of this study. In my experience, most patients choose CLND if recommended by their surgeon. So what does this study really tell us? I believe it tells us that the experienced melanoma surgeons at Memorial Sloan-Kettering Cancer Center were very good at selecting patients who were unlikely to have nodal recurrence and/or were very likely to develop distant metastatic disease, and thus were unlikely to benefit from CLND. It does not tell us that CLND is of no value.

The authors cite population studies that indicate that only $50-69 \%$ of melanoma patients in the United States who have a tumor-positive SLN undergo CLND. So why are surgeons so reluctant to perform CLND these days? To address this question, we must consider the reasons for performing CLND. There are two goals: regional disease control and cure. The patients who undergo CLND for a tumor-positive SLN fall into three camps: (1) those who do not need CLND because they do not have, and never will have, additional nodal disease; (2) those who will not benefit from CLND because they are going to die of distant metastatic disease or because their disease will recur in the regional nodes, despite the best possible lymph node dissection; and (3) those who have nonsentinel node metastases for whom CLND will prevent regional nodal recurrence and, in some cases, result in cure. The third camp undoubtedly represents the smallest group. The article by Kingham et al. implies that the third camp does 
not even exist, but I suggest that the preponderance of evidence, imperfect as it may be, suggests that regional lymphadenectomy provides regional disease control for most and cures some fraction of patients. The problem is that until we are able to predict accurately which patients belong to each of these three camps, we are left with a decision to perform potentially morbid lymphadenectomy knowing that it can only benefit a minority of patients.

The question of whether CLND improves disease-free and overall survival is the subject of the ongoing Multicenter Selective Lymphadenectomy Trial (MSLT) II, conducted by Dr. Donald Morton and colleagues. Kingham et al. conclude, however, that until the results of MSLT II are available, "nodal observation may be a reasonable alternative to the informed patient who does not want to participate in MSLT II or who does not have access to participation in MSLT II." I would suggest that this is too bold a conclusion to draw from a retrospective study of 37 patients that has the limitations described above.

\section{REGIONAL DISEASE CONTROL}

First, what is the risk of regional nodal recurrence if CLND is not performed? In two large prospective randomized trials, the Sunbelt Melanoma Trial and the MSLT I trial, the rate of positive nonsentinel nodes among patients who underwent CLND for a tumor-positive SLN was $16 \% .^{2}$ In a retrospective multi-institutional study by Wong et al. of 134 SLN-positive patients who did not undergo CLND, regional nodal recurrence was a component of first recurrence in $15 \%$ of patients. ${ }^{3}$ Overall, $41 \%$ of patients experienced regional nodal metastasis as a component of their recurrence; undoubtedly some of these patients eventually underwent therapeutic lymphadenectomy for control of regional nodal disease as well. Wong et al. reported a statistically insignificant trend toward decreased nodal recurrence-free survival in the group of patients who did not undergo CLND but no difference in overall survival compared to a contemporary series of patients who underwent CLND. ${ }^{3}$ Therefore, we can reasonably conclude from these data that the risk of developing clinically apparent and meaningful regional nodal recurrent disease as the first site of recurrence is at least $15 \%$; the fact that only $5 \%$ of patients in the study of Kingham et al. developed nodal metastasis as the first site of recurrence is likely not generalizable to the entire population of patients with tumor-positive SLN. ${ }^{1}$

Next, we must ask the question: does CLND for patients with a tumor-positive SLN improve regional disease control? In MSLT I, the rate of regional nodal recurrence after CLND was $4.2 \% .^{2}$ In the Sunbelt Melanoma Trial, it was $4.9 \%$ (unpublished data). This is much lower than the $15 \%$ rate of regional nodal recurrence as a site of first metastasis and $41 \%$ overall regional nodal recurrence rate in the study by Wong et al. ${ }^{3}$ In retrospective studies of therapeutic lymphadenectomy for clinically detectable (palpable) metastases, the rate of regional nodal recurrence ranges from 14 to $52 \%$ overall, and from 31 to $63 \%$ in high-risk groups with extracapsular extension, multiple positive nodes, nodal metastasis $\geq 3 \mathrm{~cm}$ in size, or cervical nodal basin location. ${ }^{4}$ On the basis of these comparisons of data from prospective and retrospective data, which have obvious limitations, one would conclude that CLND for patients with tumor-positive SLN is an excellent strategy for achieving regional disease control. Although some have suggested that patients with a tumor-positive SLN who do not undergo CLND can be followed by ultrasound examination of the regional nodal basin to detect nodal disease before it becomes bulky and difficult to control, this strategy remains unproven. Those of us who are called upon try to achieve regional disease control once it has been lost understand the nature of the intense pain and suffering it can cause. Hence, achieving regional disease control is a laudable surgical goal; we as surgeons should remember our solemn obligation to achieve it. Until final results of MSLT II are available, we will not be able to determine with greater-level evidence the effect of CLND on regional disease control. Until that time, the best evidence we have suggests that CLND is effective at achieving regional disease control in most patients.

\section{CURE}

Surgeons, by definition, are locoregional disease control specialists. All we can do is resect the cancer, wherever we may find it, locally or regionally. To the extent that we cure any patients, it is because they do not already have distant metastatic disease at the time we resect their local and regional disease. Resection of stage IV melanoma, when possible, also results in 5-year survival rates of up to $40 \% .^{5}$ Do we cure patients with regional nodal metastases by lymphadenectomy? The answer is a resounding yes. Even before the advent of SLN biopsy, we cured approximately one-third of patients with regional nodal disease by therapeutic lymphadenectomy. Given that there are no universally accepted effective adjuvant therapies that improve overall survival (even high-dose interferon alfa- $2 b$ therapy is controversial), and given that there are no systemic therapies for stage IV melanoma that have been shown to improve overall survival, surgical resection of all sites of disease is still the best thing we can do for melanoma patients with every stage of disease.

MSLT I (which randomized patients to SLN biopsy vs. no SLN biopsy) showed no benefit in terms of overall 
survival, likely because only a minority of patients (16\%) had tumor-positive SLN and could be potentially helped by removal of regional lymph nodes. ${ }^{2}$ However, considering only the node-positive subgroups, the 5-year survival rate for patients with tumor-positive SLN who underwent CLND was 72.3 vs. $52.4 \%$ for patients who did not undergo SLN biopsy and who developed palpable nodal disease (hazard ratio .51; $95 \%$ confidence interval, .32-.81; $P=.004)$. Similarly, in the Sunbelt Melanoma Trial, the 5 -year overall survival rate for patients with tumor-positive SLN who underwent CLND was $67 \%$ (unpublished data). Therefore, we cure most patients with stage III disease these days by early detection and aggressive surgical resection of nodal metastases. Whether or not we cure the same number of patients if we eliminated routine CLND remains to be seen. However, it is predictable that even if MSLT II (which randomizes patients with a tumor-positive SLN to CLND vs. no CLND) demonstrates a difference in survival, it will be quite small, because only $16 \%$ of patients are expected to have nonsentinel node metastases and could potentially be helped by lymphadenectomy.

\section{WHY DO SURGEONS NOT WANT TO PERFORM CLND ANYMORE?}

Yes, I have had some patients who chose not to undergo CLND, and in most of those cases, I influenced their decision. Usually this was in older patients with other comorbidities, for whom a $15 \%$ or so risk of regional nodal recurrence was less than their risk of dying of other problems within the next few years. Why did I even perform SLN biopsy for these patients? Because, for patients with high-risk primary melanomas, SLN biopsy alone does provide a measure of regional disease control. Most patients with tumor-positive SLN (84\% from MSLT I and Sunbelt Melanoma Trial data) have no additional nodes detected via CLND. We must recognize, however, that routine histopathology of nodes in the CLND specimen may miss small foci of metastatic disease that may become clinically apparent at a later date. Nevertheless, most patients will not benefit from CLND. Given the risk/benefit ratio, I think it is sometimes appropriate to suggest that CLND should be avoided. However, this is only in a small fraction of patients with extenuating circumstances. Most patients, however, do not want to leave cancer behind, and they want to do everything possible to prevent recurrence. For which other diseases do we deliberately perform less than an R0 resection when it easily can be achieved? How is this different from what surgical oncologists do for other diseases? I would argue that the risk/benefit ratio for CLND for melanoma is more favorable than that of radical resections for pancreatic cancer, esophageal cancer, locally advanced stomach cancer, etc. Even a $15 \%$ risk of nodal recurrence (which is the minimum estimate I would use) is too high for most patients.

Surgeons do not want to perform and patients do not want to undergo CLND because of the morbidity of these procedures. Until such time as we have an accurate way to determine which SLN-positive patients have a minimal risk of nonsentinel node metastases, it is difficult to balance the benefits and risks of lymphadenectomy when only a minority of patients can potentially benefit from this procedure. However, in a study comparing patients who underwent inguinal lymph node dissection for a tumorpositive SLN versus palpable nodal metastases, Sabel et al. showed that patients with palpable nodal disease had a significantly greater number of involved nodes $(3.0 \mathrm{vs}$. $1.96, P=.0013)$, more often had more than four involved nodes ( 29 vs. $9 \%, P<.001)$, and had a greater incidence of extranodal extension $(47$ vs. $5 \%, P<.001){ }^{6}$ An underappreciated reason to perform CLND for positive SLN, as opposed to waiting to see if the patients develop clinically evident disease, involves the role of adjuvant radiotherapy. In many centers, despite limited data to support its use, the finding of involved nodes of $>3 \mathrm{~cm}$ in size, multiple tumorpositive nodes, or extracapsular extension is an indication for adjuvant radiotherapy. ${ }^{4}$ There can be no question that radiotherapy to regional nodal basins adds additional morbidity and contributes to lymphedema. In the study of Sabel et al., wound complications ( 28 vs. $14 \%, P=.02$ ) and lymphedema (41 vs. $24 \%, P=.025$ ) were significantly greater among patients with palpable nodal disease versus those with a positive SLN. However, regional nodal recurrence was not significantly greater in patients with clinically palpable disease (13 vs. $9 \%, P=$ not significant), although the authors note that this result was possibly the result of the significantly greater rate of distant recurrence (49 vs. $18 \%, P<.001)$ and death $(48$ vs. $21 \%$ ) in patients with palpable disease versus a positive SLN, respectively. In virtually all cases, regional nodal recurrence after lymphadenectomy was associated with development of distant metastatic disease. ${ }^{6}$ Given the fact that the morbidity, at least for groin dissection, is significantly greater for patients who undergo therapeutic lymphadenectomy for palpable disease versus CLND for microscopically positive SLN, is it not perhaps a better strategy to perform CLND when we find tumor-positive SLN? ${ }^{6}$

How morbid is regional lymphadenectomy anyway? Is it as bad as we think? A study by de Vries et al. that examined quality of life (QoL) in melanoma patients who underwent SLN biopsy alone (with tumor-negative nodes) or SLN biopsy plus CLND provides some intriguing results. ${ }^{7}$ The study included patients with axillary and inguinal SLN biopsy and CLND. Surprisingly, the overall group of patients reported better overall QoL than a 
German norm group. Furthermore, this study did not confirm the hypothesis that patients in the CLND group had lower QoL scores than patients who only underwent SLN biopsy. Interestingly, the group that underwent axillary lymphadenectomy reported greater problems than those who underwent inguinal lymph node dissection. ${ }^{7} \mathrm{We}$ all know that lymphadenectomy is associated with morbidity, including lymphedema, but this study suggests that overall, the effect on QoL is not clinically meaningful-at least, it is not as devastating as some of us imagine. The unanswered question is whether, to patients, the utility of preventing nodal recurrence exceeds the potential negative impact of lymphadenectomy on QoL. Given these data, the choice between CLND and leaving behind nodal disease that will become clinically apparent in at least $15 \%$ of patients seems easier.

\section{FINAL THOUGHTS}

Would we cure the same number of patients if we did not perform CLND? Perhaps, but do we want to make this assumption on the basis of nonrandomized retrospective data subject to selection bias? I don't. The study of melanoma is rife with examples of nonrandomized dogma that evaporates in the face of randomized controlled trials. If not for randomized trials, we would still be performing 5$\mathrm{cm}$-margin-wide local excisions, elective lymph node dissections, adjuvant hyperthermic limb perfusions, routine biochemotherapy for patients with stage IV disease, and we would still be conducting interminable phase II studies of a variety of melanoma vaccines that have since been shown to be ineffective, if not harmful. Perhaps the conclusions I draw by comparison of randomized, nonrandomized, and retrospective data about the value of CLND are similarly flawed; I hope MSLT II will tell us the answer. Until then, the elusive truth remains uncertain-but an excellent topic for editorialists.

\section{REFERENCES}

1. Kingham TP, Panageas KS, Ariyan CE, et al. Outcome of patients with a positive sentinel lymph node who do not undergo completion lymphadenectomy. Ann Surg Oncol. (in press).

2. Morton DL, Thompson JF, Cochran AJ, et al. Sentinel-node biopsy or nodal observation in melanoma. $N$ Engl J Med. 2006;355:1307-17.

3. Wong SL, Morton DL, Thompson JF, et al. Melanoma patients with positive sentinel nodes who did not undergo completion lymphadenectomy: a multi-institutional study. Ann Surg Oncol. 2006;13:809-16.

4. Guadagnolo BA, Zagars GK. Adjuvant radiation therapy for highrisk nodal metastases from cutaneous melanoma. Lancet Oncol. 2009;10:409-16.

5. Martinez SR, Young SE. A rational surgical approach to the treatment of distant melanoma metastases. Cancer Treat Rev. 2008;34:614-20.

6. Sabel MS, Griffith KA, Arora A, et al. Inguinal node dissection for melanoma in the era of sentinel lymph node biopsy. Surgery. 2007; 141:728-35.

7. de Vries M, Hoekstra HJ, Hoekstra-Weebers JE. Quality of life after axillary or groin sentinel lymph node biopsy, with or without completion lymph node dissection, in patients with cutaneous melanoma. Ann Surg Oncol. 2009;16:2840-7. 